This preprint was published:

Kendall-Tackett, K., \& Becker-Blease, K. (2004). The importance of retrospective findings in child maltreatment research. Child Abuse \& Neglect, 28(7), 723-727.

http://dx.doi.org/10.1016/j.chiabu.2004.02.002

Child Abuse and Neglect

2004, Vol. 28, 723-727

\title{
The Importance of Retrospective Findings in Child Maltreatment Research
}

\author{
Kathleen Kendall-Tackett, Ph.D. and Kathryn Becker-Blease, Ph.D. \\ Family Research Laboratory and Crimes against Children Research Center \\ University of New Hampshire
}

In two recent reports, Widom and colleagues (Raphael, Widom, \& Lange, 2001; Widom, Weiler, \& Cottler, 1999) found that prospective findings differed from retrospective findings in the same sample. In both studies, the prospective findings showed no relationship between maltreatment and two outcomes--chronic pain or substance abuse--but did find a relationship between maltreatment and these outcome variables when subjects reported retrospectively. The subjects identified prospectively all had histories of maltreatment documented during childhood. They were compared to a matched non-abused control group. In contrast, the retrospective findings were based on questionnaire data when the subjects were adults, 20 years later. The questionnaire asked subjects in both the maltreated and control groups if they had experienced any of the abusive or neglectful acts before age 12. When subjects identified their abuse status retrospectively, there were strong relationships between maltreatment and pain (Raphael et al., 2001), and maltreatment and substance abuse (Widom et al., 1999).

The results of these two studies raise a very important methodological issue. Can the maltreatment field trust data collected retrospectively? Dube and her colleagues from the Adverse Childhood Experiences (ACE) study (Dube, Williamson, Thompson, Felitti, \& Anda, 2004) say that retrospective findings are trustworthy. The ACE study is a large epidemiological study that examines the health impact of adverse childhood events on the health of adults in an HMO population. In the current study, they used data from 658 participants who completed the ACE study on two separate occasions, approximately a year apart. They found some mild differences in the two reports, but overall retrospective reports of adverse childhood events were stable over time.

For child maltreatment researchers, the reliability of retrospective findings is no mere methodological quibble. Much of what we know about the long-term effects of childhood abuse comes from retrospective studies. But what about the prospective findings? Depending on the research question, prospective studies can provide a number of significant advantages over retrospective designs. Researchers often consider prospective designs to be more rigorous, with less biased sample selection and better measurement of the natural history of a phenomenon. For studies of certain outcome variables, it is the most appropriate design. Williams' (1994) study of amnesia in abuse survivors is a good example. The subjects in that study all had sexual abuse experiences documented when they were children. Twenty years later, they were asked about events that had happened in their lives. The interviewers did not mention the previous documentation. More than a third of these women seemed to have no memory for their 
abuse experiences. If the study had depended on their self-identification, these women would have been overlooked.

Given the strengths of prospective data, does this mean that we should now disregard findings from retrospective studies? Not necessarily.

\section{Why the Field Needs Retrospective Data}

In contemplating the larger question of retrospective vs. prospective data, researchers should not assume that prospective is "better." To do so would, in fact, skew knowledge about child maltreatment. For example, prospective designs of the sequelae of past abuse require that abuse survivors be identified as children and then followed. The problem is, that unlike other subject populations (e.g., women who have recently given birth), researchers cannot simply identify and follow them. Professionals who discover abuse must report it to law enforcement and/or child protection agencies, which are ethically bound to intervene in some way. Once the children are identified, action must be taken. Therefore, this group of identified children has a higher likelihood of receiving some type of intervention or treatment during childhood compared with children who are abused but not identified. Intervention, even if inept, may have halted the biological and psychological processes that could have led to serious sequelae.

There are two other issues related to the importance of retrospective findings: 1 ) prospective designs may miss cases, and 2) unreported abuse may be more severe. These issues are described in more detail below.

\section{Prospective Designs May Miss a Substantial Number of Cases}

Child maltreatment, by its very nature, takes place under a cloak of secrecy. Prospective designs alone would miss many subjects because a high percentage of adult survivors never reported their abuse to anyone. (In one of the first author's early studies, $82 \%$ of 365 survivors of sexual abuse had never reported their abuse to law enforcement or state agencies [Kendall-Tackett, 1991].) The results of Raphael et al. (2001), which examined the relationship between childhood abuse and chronic pain in adults, even suggest this discordance. When interviewed 20 years later, the number of subjects in each maltreatment category were substantially higher in the self-report vs. documented conditions, meaning that some adults in the control group were reporting abuse experiences as well. For example, there were 96 subjects with documented cases of sexual abuse, but 291 who self-reported sexual abuse. Similarly, there were 100 subjects with documented physical abuse, and 610 with self-reported physical abuse.

For this reason, prospective findings cannot be solely relied upon since subjects who were identified as children are probably not representative of abuse survivors as a whole. Subjects in retrospective vs. prospective designs may, in fact, be two distinct subgroups within the population of adult survivors. Therefore, the findings might differ because the subjects differ as opposed to the different directionalities of the research. 


\section{Unreported Abuse May Have Been More Severe}

When abuse is identified during childhood, in most cases, it stops. But when no one knows about ongoing abuse, it can continue, and may even escalate. One way that researchers measure severity of abuse is the duration of the experience. Abuse that goes on for a longer time is often considered more severe. In addition, secretive abuse may have included more shame and isolation. The increased severity of these experiences, and the potentially higher levels of shame and isolation, could also account for more symptoms in retrospective studies. For example, in one retrospective study of pain, severity of abuse was related to severity of symptoms (Drossman, Li, Leserman, Toomey, $\& \mathrm{Hu}, 1996)$. When severity of abuse was not taken into account, the amount of variance in health functioning accounted for by abuse history was fairly small, ranging from $1 \%$ to $10 \%$. However, when only patients with severe abuse were considered, abuse history accounted for $63 \%$ of the variance for days in bed, and $74 \%$ for number of physician visits.

\section{Some Ways to Verify Findings from Retrospective Studies}

Although we favor including retrospective findings, Widom et al. and Raphael et al.'s findings give us pause. To increase confidence in retrospective findings, it is helpful to corroborate our findings if at all possible. Here are some suggested questions to ask.

\section{Is the Link Plausible?}

This is really an argument for face validity. Does this finding make sense? Clinicians treating adult survivors can often answer this one. Are they seeing the outcome variable of interest in their patients who have survived childhood abuse?

The research on the physiology of maltreatment can also provide support for plausibility. Given what researchers know about the physiology of maltreatment, could there be a biological mechanism that supports these symptoms? Let's take the example of chronic pain. Abuse survivors often have aberrant levels of stress hormones and neurotransmitters, and their sleep patterns are often altered as well (Kendall-Tackett, 2003; Meagher, 2004; Teicher, 2002). The state of research in this field is still new enough that researchers cannot definitively state that abuse and trauma actually causes chronic pain, but the relationship between child maltreatment and pain is consistent with the biological evidence.

\section{Are There Similar Findings in Analogous Populations?}

Another issue that is often raised in describing the discrepancy between prospective and retrospective findings is memory bias. In other words, are subjects misremembering childhood events? For patients who are now having chronic pain, are they now painting their childhood experiences as worse than they really were? If subjects in pain/abuse studies were adults when the trauma occurred, their reports would be less likely to suffer from distortions in memory. If adults had the same types of symptoms 
that children had, it might suggest that more than memory was at work (e.g., possible biological processes at work whereby traumatic events increase pain). There is some evidence to support this view.

In one of the original studies on pain and abuse, Harrop-Griffiths and colleagues (1988) found a similar pattern between those whose abuse happened in childhood and those women whose abuse occurred in adulthood. Patients who had pelvic pain were significantly more likely to have been victims of either childhood (64\% vs. $7 \%$ ) or adult sexual abuse ( $48 \%$ vs. $13 \%$ ) than the comparison group.

Similarly, in a large community sample from Los Angeles, Golding (1994) found that women who had been sexually assaulted at any time in their lives were significantly more likely to report chronic pain in many different parts of their bodies. Sixty-percent of the assaulted women reported six or more pain symptoms compared with $36 \%$ of the non-assaulted women.

In a more recent study, Golding (1999) found that in her meta-analysis of five community samples, past sexual assault was related to headaches. This was true for both sexual assault in childhood and sexual assault in adulthood, but the odds ratios were higher for childhood assault. Golding's studies are also notable because the samples were drawn from community populations, not those in treatment for chronic pain (whose pain levels tend to be higher).

Another way to examine this issue is to ask whether common sequelae of abuse, such as PTSD, are also related to increased pain symptoms. One study of combat veterans with PTSD did find a relationship. In a sample of 921 older veterans (mean age $=65$ years), Schnurr and Spiro (1999) found that chronic pain was highly correlated with PTSD. The veterans with PTSD also reported a lower level of overall physical and mental health than veterans who did not have PTSD. All the subjects in this study experienced trauma as young adults. (This study did not ask about childhood trauma, but another study of veterans [Bremner et al., 1993] found that men who had been physically abused as children were more prone to combat-related PTSD. Childhood abuse did not account for all combat-related PTSD, however.)

\section{Toward Future Studies}

Widom et al.'s studies raise some other interesting questions. One area to explore is whether patients' self-identification as abuse survivors, rather than those who appear to have no memory for past events or do not call themselves survivors, increases symptoms of past abuse (C. Widom, personal communication, July, 2002). Specifically, is there something about the process of identifying oneself as an abuse survivor that relates to the reporting of symptoms? Are people with severe symptoms more (or less) likely to identify themselves as abuse survivors? Does dissociation have any impact on those who do not identify themselves as abuse survivors, even when they are? And could dissociation influence somatic awareness of patients and their willingness to report pain or other symptoms (Haven \& Pearlman, 2004)? 
Interestingly, in a study of the pain syndrome fibromyalgia, researchers compared two groups: those who were receiving treatment for fibromyalgia (the patient group), and those who met criteria for fibromyalgia, but were not in treatment (the non-patient group). When both groups were asked about their abuse experiences, there was a higher percentage of abuse survivors in the patient vs. non-patient group (Aaron et al., 1997). Investigators found that $54 \%$ of the patient group had been physically or sexually abused, compared with $36 \%$ of the non-patient group. These findings raise some interesting issues for future studies. One possibility is that abuse survivors who seek treatment may be more likely to label both their past experiences as abuse and to seek treatment for physical health concerns. Or patients who seek treatment may be sicker than those who do not because they have experienced more severe abuse.

\section{Conclusions}

The discrepancy between prospective and retrospective findings has highlighted some important issues that need to be considered in future studies of symptomatology in adult survivors. Prospective findings are valuable, but retrospective findings measure a segment of the population of adult survivors missed by prospective studies. In short, there is room for both retrospective and prospective findings. Both types of research provide a glimpse into the long-term sequelae of childhood abuse, and the maltreatment field would lose valuable information if retrospective findings were discarded.

\section{References}

Aaron, L.A., Bradley, L.A., Alarcon, G.S., Triana-Alexander, M., Alexander, R.W., Martin, M.Y., \& Alberts, K.R. (1997). Perceived physical and emotional trauma as precipitating events in fibromyalgia. Arthritis \& Rheumatism, 40, 453-460.

Bremner, J.D., Southwick, S.M., Johnson, D.R., Yehuda, R., \& Charney, D.S. (1993). Childhood physical abuse and combat-related posttraumatic stress disorder in Vietnam veterans. American Journal of Psychiatry, 150, 235-239.

Drossman, D.A., Li, Z., Leserman, J., Toomey, T.C., \& Hu, Y.J.B. (1996). Health status by gastrointestinal diagnosis and abuse history. Gastroenterology, 110, 9991007.

Golding, J.M. (1994). Sexual assault history and physical health in randomly selected Los Angeles women. Health Psychology, 13, 130-138.

Golding, J.M. (1999). Sexual assault history and headache: Five general population studies. The Journal of Nervous and Mental Disease, 187, 624-629.

Harrop-Griffiths, J., Katon, W., Walker, E., Holm, L., Russo, J., \& Hickok, L. (1988). The association between chronic pelvic pain, psychiatric diagnoses, and childhood sexual abuse. Obstetrics and Gynecology, 71, 589-593. 
Haven, T.J., \& Pearlman, L.A. (2004). Minding the body: The intersection of dissociation and physical health in relational trauma psychotherapy. In K.A. Kendall-Tackett (Ed.), The health consequences of abuse in the family: A clinical guide for evidence-based practice (pp. 215-232). Washington, DC: American Psychological Association.

Kendall-Tackett, K.A. (2003). Treating the Lifetime Health Effects of Childhood Victimization. Kingston, NJ: Civic Research Institute.

Kendall-Tackett, K.A. (1991). Characterisitics of abuse that influence when adults molested as children seek treatment. Journal of Interpersonal Violence, 6, 486493.

Meagher, M.W. (2004). Links between traumatic family violence and chronic pain: Biopsychosocial pathways and treatment implications. In K.A. Kendall- Tackett (Ed.), The health consequences of abuse in the family: A clinical guide for evidence-based practice (pp. 155-177). Washington, DC: American Psychological Association.

Raphael, K.G., Widom, C.S., \& Lange, G. (2001). Childhood victimization and pain in adulthood: A prospective investigation. Pain, 92, 283-293.

Schnurr, P.P., \& Spiro, A. III. (1999). Combat disorder, posttraumatic stress disorder symptoms and health behaviors as predictors of self-reported physical health in older veterans. Journal of Nervous and Mental Disease, 187, 353-359.

Teicher, M.H. (2002). Scars that won't heal: The neurobiology of child abuse. Scientific American, March, 68-75.

Widom, C.S., Weiler, B.L., \& Cottler, L.B. (1999). Childhood victimization and drug abuse: A comparison of prospective and retrospective findings. Journal of Consulting and Clinical Psychology, 67, 867-880.

Williams, L.M. (1994). Recall of childhood trauma: A prospective study of women's memories of child sexual abuse. Journal of Consulting and Clinical Psychology, 62, 1167-1176. 